\title{
Bilateral, posterior parietal polymicrogyria as part of speech therapy work-up
}

\author{
J L Höll, MB ChB, FFRad (D)SA \\ Department of Radiology, Pretoria Academic Hospital, University of Pretoria \\ I Smuts, BSc, MMed (Paed) \\ Department of Paediatrics and Child Health, Pretoria Academic Hospital, \\ University of Pretoria
}

Congenital bilateral perisylvian syndrome (CBPS) was traditionally associated with pseudobulbar palsy, cognitive deficits and epilepsy. However studies have found that the clinical spectrum of CBPS is much broader including patients without epilepsy, but with serious developmental language disorder. Magnetic resonance imaging (MRI) has been associated with either diffuse polymicrogyria around the entire extent of the sylvian fissure or in the posterior aspects of the parietal regions, in which case it is called posterior parietal polymicrogyria.

This article discusses the possible embryological origin of these lesions, the MRI findings using specific protocols and the ability of thinslice MRI 1.5 Tesla units to make the diagnosis of bilateral posterior parietal polymicrogyria in a child with speech pathology.

\section{Introduction}

Polymicrogyria is an abnormality in the cortical development where the normal development of the cortex is interrupted in the late stages of neuronal migration and cortical organisation. ${ }^{1}$ This interruption results in abnormal development of the deep layers of the cerebral cortex and production of multiple small gyri and small plications ${ }^{2}$ on the cortical surface.

Ranges of histological appearances are seen in polymicrogyria but basically all have a variation in derangement of the normal 6-layer lamination of the cortex. In short there are 2 basic variations seen, notably layered and unlayered. In the unlayered variation the neurons descending to form the outer layer of cortex are halted in their migration. The layered variant shows the distant layers deranged due to possible destructive lesions originating prenatally, e.g. toxoplasmosis or cytomegalovirus $(\mathrm{CMV})$. It has also been diagnosed in fetuses of mothers with carbon monoxide poisoning at $20-24$ weeks' gestation. ${ }^{2}$ Three chromosomal mutations (Xq28, 16g, 12.2.21) have also been described. ${ }^{3}$

On studying the literature, no difference in neurological ${ }^{1,2}$ manifestation on MRI could be detected between any of the pathologies described above.

Affected patients may present at any age, but there is some correlation between the extent of cortical involvement and severity of the clinical presentation. ${ }^{1}$

These malformations are most common around the sylvian fissure particularly in the posterior aspect of the fissure but can also be diffuse, focal, unilateral, bilateral or asymmetrical, and have been described in all areas of the cortex, from the frontal to the occipital regions.

Patients typically present with pseudobulbar palsy (80 - 90\%), oropharygeal dysfunction (90 - 100\%), dyarthria (100\%), epilepsy (80 - 90\%) and mental retardation (50 - 80\%). Some patients present in infancy or early childhood with developmental delay, poor parallel function, hypotonia, arthrogryposis and mental defects. ${ }^{1}$

It is interesting to note that the epileptogenic focus is typically not within the dysplastic context itself but in the cortex adjacent to the paramicrogyral zone. ${ }^{4}$

In the imaging of polymicrogyria, use of the 1.5 Tesla unit is recommended with thin sections less than $5 \mathrm{~mm}$, preferably $2-3 \mathrm{~mm}$. The reason is that the cortical surface may have an irregular bumpy appearance or be paradoxically smooth due to outer cortical layers fusing over the micro-sulci. As expected, the dysplastic cortex typically shows an irregular inner and outer cortex surface and may have the appearance of pachygyria or may even be normal in thick sections. Evaluation in 3 planes is necessary to detect irregularities of the grey/white matter junction, which is often the only evidence of dysplastic ${ }^{1,4}$ brain.

Multiple programmes are used by different institutions but the tendency in South Africa is for paediatric neuroimaging to include T1weighted short TR and short TE, T2-weighted TR and long TE, and very long Flair TR (9 000)/long TE with contrast if needed. ${ }^{1,5}$

The case in this study is a very good example of what we have been doing in this time. The differential diagnosis ${ }^{6}$ or other possibilities include: (i) microlissencephaly and pachygria or incomplete lissencephaly; (ii) malformations secondary to inborn errors of metabolisma, e.g. mitochondrial and pyruvate metabolism disorders, Zellweger syndrome; (iii) hemi-megalencephaly; and (iv) congenital CMV, as noted in the introduction.

\section{Case report}

A male child of 3 years and 7 months was referred to the Pretoria Academic Hospital Speech Therapy and Audiology Department. The parents' main concern was the child's difficulty eating solid food as well as his low body weight. The secondary complaint was severe delay in speech development.

The following information and clinical symptoms and signs were observed and noted during the paediatric neurological assessment.

The mother had had flu-like symptoms at 6 months of gestation but the rest of the pregnancy was uneventful. The birth weight was 2.6 $\mathrm{kg}$ after an uneventful caesarean section. At birth it was noted that the infant was able to suck. He had a history of recurrent episodes of tonsillitis. His 3 siblings were all normal, but the father had experienced some delays in speech development as child. He had normal speech as an adult. The paternal aunt had been treated for epilepsy.

Speech therapy reported that the child communicated via gestures. According to the parents he was able to say a few 2-syllable words, but no use of words was observed during the assessment. He showed signs of frustration when he was misunderstood. However, certain precursors to communication, e.g. the intention to communicate, eye contact and turn-taking were observed. The child was also able to follow simple 


\section{CASE REPORT}

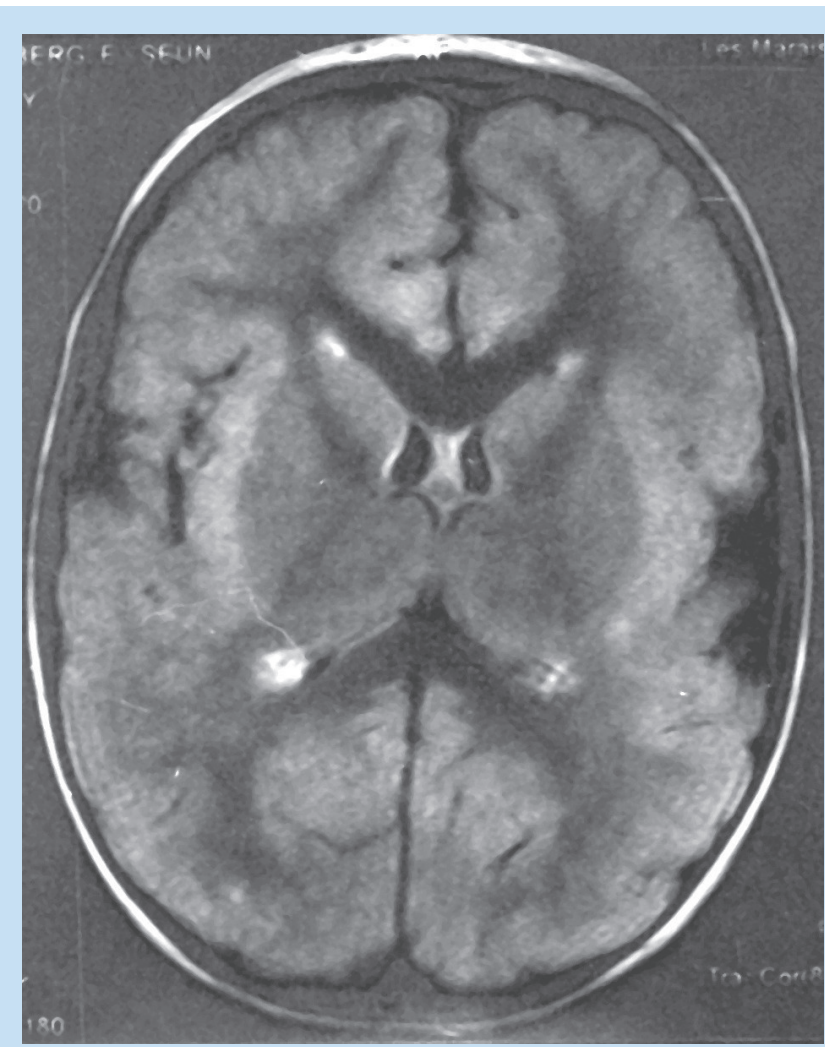

Fig.1. Axial FLAIR sequence. Note the cortical defect in the perisylvian region, more prominent on the left side.

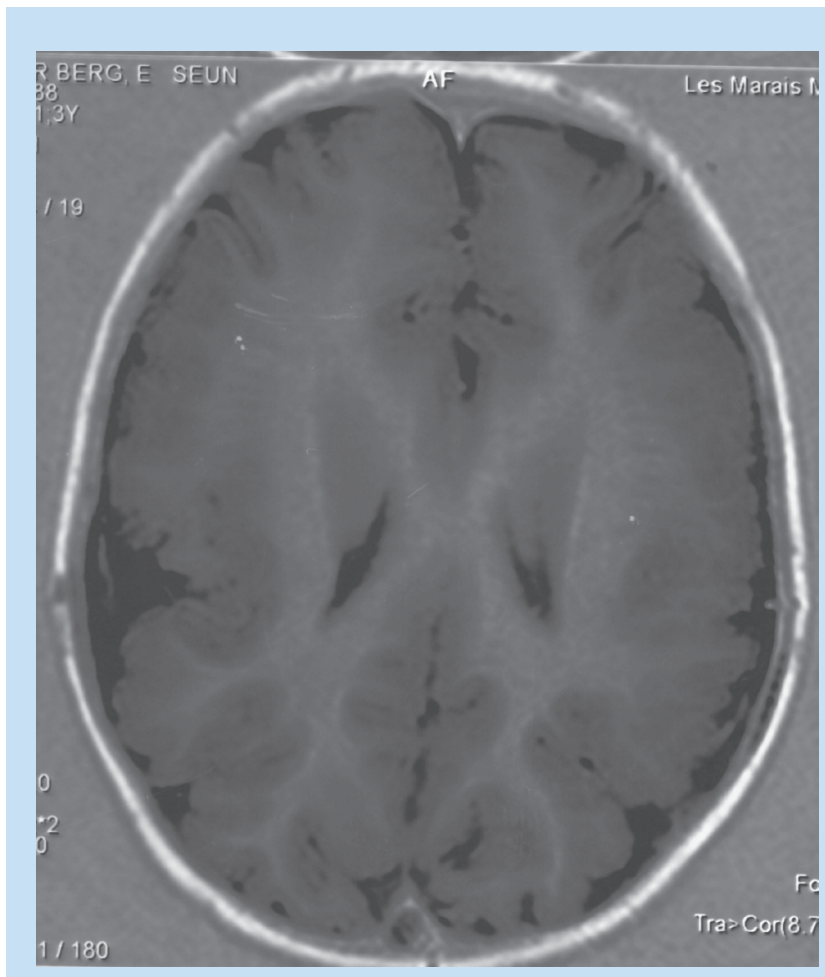

Fig. 2. Axial T1-weighted image with inversion recovery sequence. Irregular cortical surface with small and multiple gyri. Note the loss of cortex.

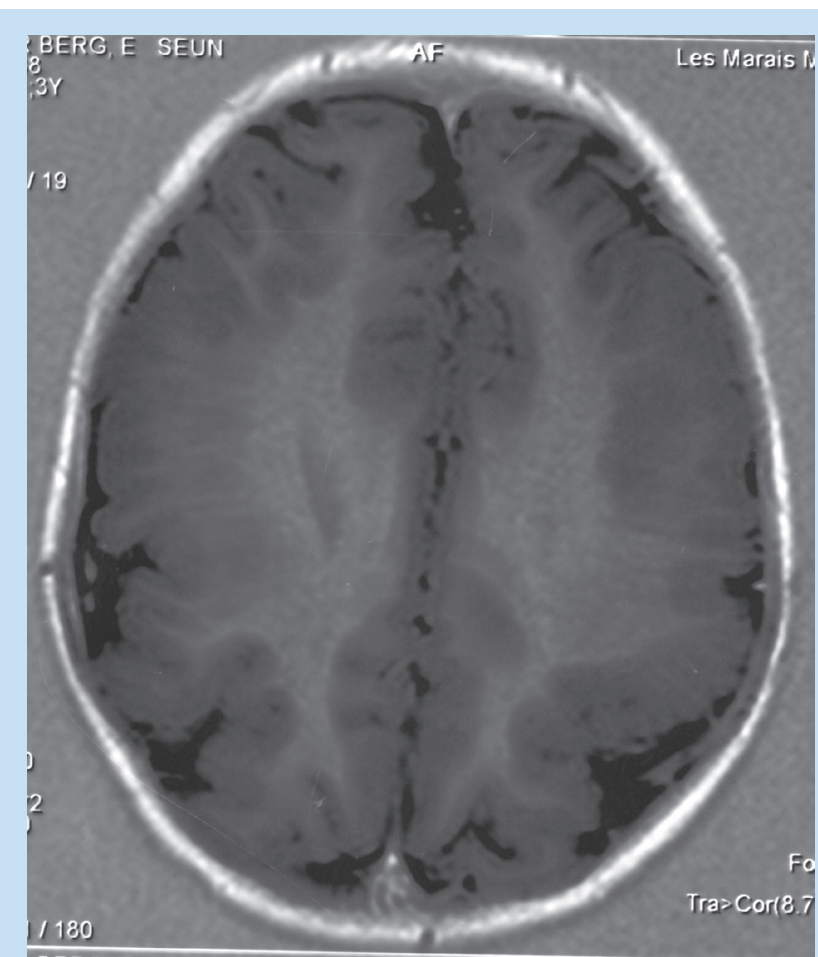

Fig.3. Axial T1-weighted image with inversion recovery sequence. Some cortical loss with irregular cortex, noted in the posterior aspect of the sylvian fissure extending onto the parietal region.

instructions as well as point to objects / pictures appropriately. He also responded appropriately to sound and speech and had normal hearing on testing.

Neurological evaluation revealed a healthy boy, but growth impairment was obvious. He had symmetrical growth retardation, and weight, height and skull circumference were below the 50th percentile. No signs of malnutrition or any organomegaly was observed. Gross and fine motor development were at the level of 2-year-old child. Expressive language development was at the level of a 9 - 12 month-old child.

Good receptive abilities and the ability to follow simple instructions were noted. Significant drooling was noted but the gag reflex was present. Cranial nerve evaluation revealed no nerve palsies. During a previous examination the possibility of pseudobulbar signs was noted but during evaluation by the paediatric neurologist, motor, sensory and cerebellar systems were all within normal limits. No basal ganglia abnormalities were found. A clinical diagnosis of oral apraxia was made.

During the video fluoroscopy it was very difficult to obtain the necessary information. The boy presented with oral hypersensitivity and disliked being touched in or around his mouth. He was also unable to protrude his tongue.

During the video fluoroscopy assessment of swallowing the child did not recognise food in the mouth, no tongue movement was observed and poor control of bolus, with constant drooling was seen, indicating an inability to control saliva. After placement of a large bolus, the initiation of swallowing did occur but a part of the bolus was swallowed. Post swallow, a large amount of food bolus remained in the mouth, with coating of the tongue and bolus residue on the posterior wall of the pharynx. 
No primary or secondary aspiration was noted and the remainder of the oesophagus down to the level of the cardia and stomach was normal. A chest radiograph revealed no acute aspiration or signs of chronic aspiration.

After the video fluoroscopy MRI was done using a 1.5 Tesla magnet, using the following sequences: axial FLAIR, sagittal T1-weighted, and coronal T2-weighted images (Figs 1-3).

Axial strong T2 with inversion recovery sequence revealed an abnormal grey matter configuration seen bilaterally in the parietal regions. The changes stretched superiorly and inferiorly and were more prominent on the left side. The left temporal region was relatively smaller than the right side, with the grey matter in the left temporal region very pertinently abnormal.

The white matter on the left side was slightly thinner than on the right side.

The final conclusion was that the images supported the diagnosis of bilateral posterior parietal polymicrogyria.

\section{Conclusion}

It is suggested that children with expressive language delay, excessive drooling and failure to thrive should be assessed for polymicrogyria. It requires a multidisciplinary team approach, as illustrated in this case study. Members of the team should include at least a radiologist, paediatric neurologist, speech therapist and dietician. It has also been suggested that MRI is the most cost-effective single specific investigation in the diagnosis of polymicrogyria.

1. Barkovich AJ. Pediatric Neuroimaging. 4th ed. Philadelphia: Lippincott Williams and Wilkins, 2005: 360 $-362$

Volpe JJ. Neurology of the Newborn. 4th ed. Philadelphia: WB Saunders, 2001: 67 - 68. Jansen A, Andermann E. Genetics of the polymicrogyria syndromes. J Med Genet 2005: 42: 369 - 378.

4. Jacobs K, Kharazia A, Prince D. Mechanisms underlaying epileptogenesis in cortical malformation. Epilepsy Res 1999: 36: 165 - 188.

5. Andronikou S, Wieselthaler N, Kader E. Baby Steps in Paediatric Neuroradiology. Cape Town: South African Medical Association Health and Medical Publishing Group, 2004.

6. Osborn AG, Blaser SI, Salzman KL, Katzman GL, Provenzale J, Castillo M. Diagnostic Imaging Brain. 1st ed. Salt Lake City, Utah: Amirsys Publications 2004: 62-65.

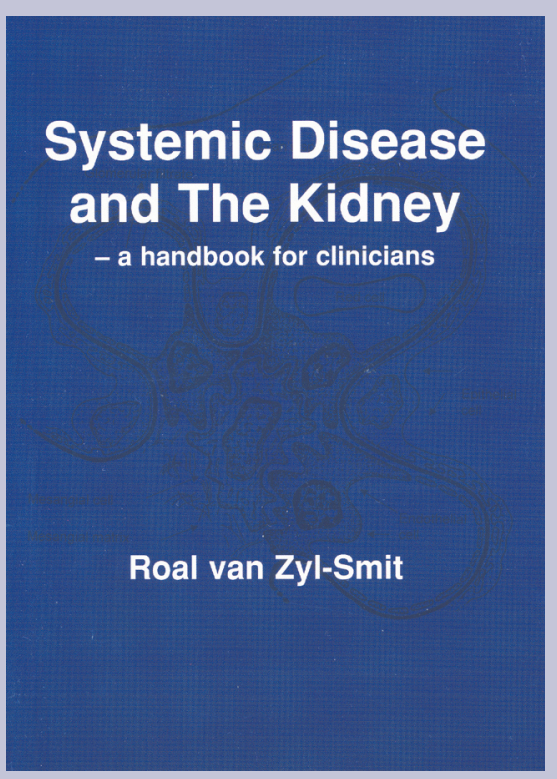

\title{
SAMA member price: $\mathrm{R} 269,00$ Non-members: $R$ 289,00
}

\author{
All prices include VAT and Postage
}

Systemic Disease and the Kidney - a handbook for clinicians - is available only through the SAMA - HMPG Bookshop, where SAMA members enjoy special discounts on all purchases.

To order: Tel: (021) 5306520 / 6551 • Fax: (021) 5314126 / 3359 • E-mail: books@samedical.org 